\title{
Synthesis and copolymerization of pentachlorophenyl acrylate monomers
}

\author{
Qin Wang ${ }^{\mathrm{a}, \mathrm{b}}$, Guan-Quan Wang ${ }^{\mathrm{a}}$, Yong-Hen Fan', Lin Zhang ${ }^{\mathrm{c}}$, and Xiao-Qi Yü* \\ ${ }^{a}$ Department of Chemistry, Key Laboratory of Green Chemistry and Technology \\ (Ministry of Education), Sichuan University, Chengdu 610064, P. R. China \\ ${ }^{b}$ Luzhou Medical College, Luzhou 646000, P. R. China \\ ${ }^{c}$ National Key Laboratory of Laser Fusion, Mianyang 621900, P. R. China \\ E-mail:xqyu@ttfol.com
}

\begin{abstract}
Pentachlorophenyl acrylate monomers were synthesized for the first time via the condensation reaction of acrylic acids with pentachlorophenol by using N,N'-dicyclohexylcarbodiimide as condensing agent. Copolymerization of the acrylate monomers with pentaerythritol tetraacrylate was studied. The preliminary results indicated that the acrylate foams can be produced on supercritical carbon dioxide drying.
\end{abstract}

Keywords: Synthesis, monomers, copolymerization, pentachlorophenyl acrylate

\section{Introduction}

Acrylates are the efficient multifunctional monomer used in foaming processes because of their rapid polymerization rates and desirable final network properties. ${ }^{1-4}$ Acrylate foams have been used extensively in the fabrication of targets for inertial confinement fusion (ICF) and laserdriven plasma physics experiments,${ }^{5-8}$ and they have been proven to be versatile foams for the in situ polymerization and the production of targets embedded with objects, ${ }^{9,10}$ shock experiments ${ }^{11}$ and for the investigation of the production of a uniform energy deposition in direct drive ICF. ${ }^{12}$ Although many achievements have been made for improving low density and sized-cell dimensions of the acrylate foams, a conspicuous urgency currently lies in making the foam not fragile, resistant to shrinking force. The foam containing a number of chlorines can promote early gelation, increase the strength significantly and meet the physical requirement with diagnostic aim such as some laser experiments involving X-ray spectrometry.$^{13-15}$

Previous reports have been focused on three different strategies for the foam containing with a number of chlorines. ${ }^{16}$ It has been proven possible that a salt solution is slowly added to the wet foam prior to freeze drying. The second route to the foams is via ion-exchange resins. 
However these two methods are only limited in physical doping process. Successful foams have been produced from polychlorostyrene by addition of hydrogen chloride or chlorine to double bonds in ethylene-type structures. Where the desired element is chlorine, it is possible to produce foams where the chlorine is chemically bound to the polymer.

We report here a novel method to prepare the acrylate monomers with chlorine by introducing successfully pentachlorophenyl group into the backbone of acrylate monomer. Copolymerization of the acrylate monomers containing chlorine with pentaerythritol tetraacrylate (PETA) is studied. The preliminary survey indicates that the acrylate foams can be produced on supercritical carbon dioxide drying and the chlorine is distributed into the foam structure all over by chemically bond method.

\section{Results and Discussion}

\section{Monomer synthesis}

The synthetic route for pentachlorophenyl (PCP) acrylate monomers 5 is shown as Scheme 1. Crystalline "complex" 3 consisting of an isourea derivative was synthesized conveniently from $N, N$ '-dicyclohexylcarbodiimide 1 (DCC) and pentachlorophenol 2 (PCPOH) in ethyl acetate according to the literature. ${ }^{17}$ The IR spectrum of $\mathbf{3}$ in solid state shows little or no absorption at $2118 \mathrm{~cm}^{-1}(-\mathrm{N}=\mathrm{C}=\mathrm{N})$ and has a strong band at $1686 \mathrm{~cm}^{-1}(\triangle \mathrm{C}=\mathrm{N}-$ ). The intermediate 3 has been used for a long time to obtain active pentachlorophenyl ester in high yield in the synthesis of peptides and polypeptides. ${ }^{18}$ As our extension of DCC methodology in synthesis of cyclic peptides, ${ }^{19}$ We found that the reaction of $\mathbf{3}$ with acrylic acids $\mathbf{4}$ can also afford monomers $\mathbf{5}$ in good yield (70-78\%). This indicates that acrylic acid makes a direct nucleophilic attack on the aromatic nucleus of the isourea derivative. The structures of monomers have been confirmed by ${ }^{1} \mathrm{H}$ NMR, ES-MS and their melting point was compared with the literature. ${ }^{20-22}$ These results are summarized in Table 1. Thus, we have synthesized the perchlorinated acrylate monomers by introducing successfully PCP group into the backbone of acrylate monomer. It was also reported for the perfluoroalkylated monomer by transetherification reaction. ${ }^{23}$ To date, the methods of preparing the acrylate monomers 5 have been reported only in the reaction of the phenol with corresponding acryloyl chlorides in the presence of triethyl amine. ${ }^{24-27}$ 


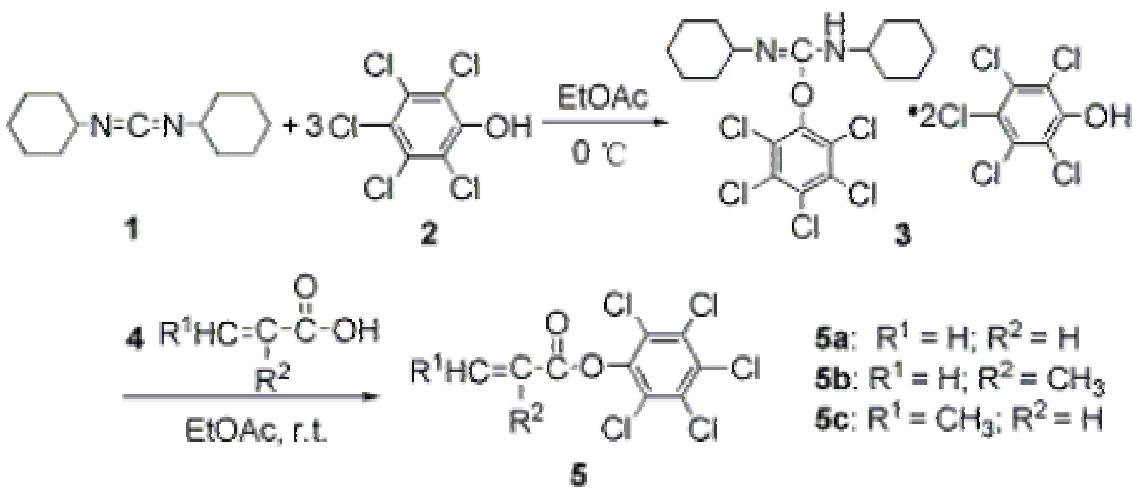

Scheme 1. Synthetic route for the active pentachlorophenyl acrylate monomers 5

Table1. The results on synthesis of active acrylate monomers.

\begin{tabular}{|c|c|c|c|c|c|c|}
\hline Monomer & $\mathrm{R}^{1}$ & $\mathrm{R}^{2}$ & ${ }^{1} \mathrm{H} \mathrm{NMR}\left(\mathrm{CDCl}_{3}, \mathrm{ppm}\right)$ & $\mathrm{MS}(\mathrm{m} / \mathrm{z})$ & Yield $^{\mathrm{a}}$ & $\mathrm{Mp}\left({ }^{\circ} \mathrm{C}\right)^{\mathrm{b}}$ \\
\hline $5 \mathbf{5 a}$ & $\mathrm{H}$ & $\mathrm{H}$ & $\begin{array}{l}\text { 6.17(d, J=10.4Hz.1H) } \\
6.36(\mathrm{dd} .1 \mathrm{H}) \\
6.72(\mathrm{~d} . \mathrm{J}=17.2 \mathrm{~Hz} .1 \mathrm{H})\end{array}$ & $\begin{array}{l}319\left(\mathrm{M}^{+}\right) \\
56(100)\end{array}$ & $78 \%$ & $\begin{array}{l}80-82 \\
(78-80)\end{array}$ \\
\hline $5 \mathbf{b}$ & $\mathrm{H}$ & $\mathrm{CH}_{3}$ & $\begin{array}{l}2.10(\text { s. } 3 \mathrm{H}) \\
5.90(\text { s. } 1 \mathrm{H}) \\
6.48(\text { s. } 1 \mathrm{H})\end{array}$ & $\begin{array}{l}331\left(\mathrm{M}^{+}\right) \\
69(100)\end{array}$ & $73 \%$ & $\begin{array}{l}89-90 \\
(91-91.5)\end{array}$ \\
\hline $5 \mathbf{c}$ & $\mathrm{CH}_{3}$ & $\mathrm{H}$ & $\begin{array}{l}2.02(\mathrm{~d}, \mathrm{~J}=6.8 \mathrm{~Hz} .3 \mathrm{H}) \\
6.09(\mathrm{~d}, \mathrm{~J}=15.2 \mathrm{~Hz} .1 \mathrm{H}) \\
7.29(\mathrm{~m}, 1 \mathrm{H})\end{array}$ & $\begin{array}{l}335\left(\mathrm{M}^{+}\right) \\
69(100)\end{array}$ & $70 \%$ & $\begin{array}{l}96-101 \\
(104-105)\end{array}$ \\
\hline
\end{tabular}

${ }^{\mathrm{a}}$ Isolated yield; ${ }^{\mathrm{b}}$ Melting point in parentheses is the literature value, see references $20-22$.

\section{Copolymerization}

In this study, the acrylate monomer $\mathbf{5}$ was used as the difunctional monomer and pentaerythritol tetraacrylate (PETA) was employed as the cross-linking agent (Scheme 2). The molar ratio of monomers 5 to PETA used for copolymerization procedure was 1 to 2 according to the desired content of chlorine about $20 \%$ in the foam which meet the physical requirement in the fabrication of targets. Dioxane was used as solvent because it was an acceptable compromise in a number of conflicting factors such as effect of chain transfer from the solvent, solubility of the monomer and the polymer and viscosity. 2,2'-Azobis(isobutyronitrile) (AIBN) was used as the thermal initiator in this copolymerization. We have ever used benzil and benzoyl peroxide as the photoinitiators, but the result despaired. ${ }^{14}$ A possible reason for the failure of photochemical initiation could be due to the presence of aromatic group in active acrylate monomer. The aromatic group can easily absorb UV light to cause the dimerization of monomer. These dimers do not break down to generate free radical and can be washed out in solvent exchange process. 


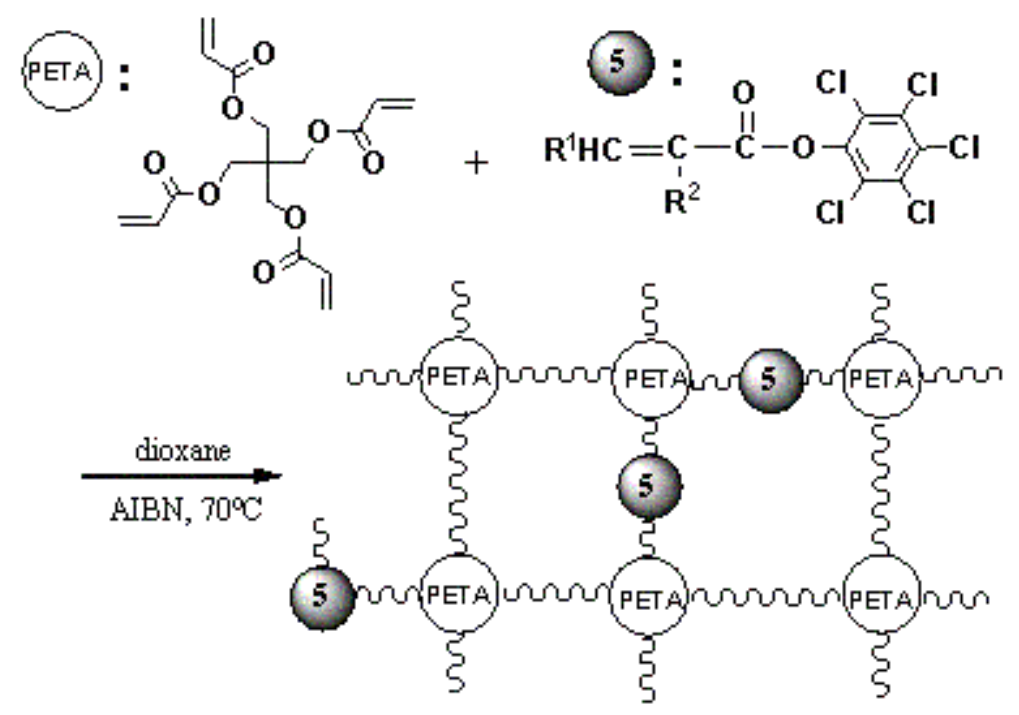

Scheme 2. Copolymerization of the monomers 5a with PETA.

The copolymerization of the monomer $\mathbf{5}$ with PETA gave a gel polymer. Viscosity was investigated as the solution property of the polymer by using ultrasonic viscometer. Figure 1 indicates that the viscosity change with the reaction time for copolymerization of the monomer 5a with PETA. Though there is no obvious change for the viscosity of solution with slight increase in the period of time from $\mathrm{A}$ to $\mathrm{B}$, carbon-carbon double bond of the monomer is broken and gradually polymerized into low-molecular polymer. Beginning with the time point $\mathrm{B}$, it is observed that the viscosity increase sharply with the increase of time until it reaches a constant value called gel point $\mathrm{C}$. This behavior is due to the rapid formation of net-gel structure by crosslinking of chemical bond in low polymers. Subsequently, the viscosity of polymer is characteristically steady and the net-structure of copolymer is further improved. The slight decrease in viscosities is attributed to a small amount of decomposition and damage of netstructure of gel after time point $\mathrm{D}$. The period of time for copolymerization of the monomers 5 with PETA is summarized in Table 2. The results indicate that there is a difference of gel rate for copolymerization of active acrylate monomers $\mathbf{5 a}, \mathbf{5 b}$ and $\mathbf{5 c}$ with PETA and the formation of gel for monomer $\mathbf{5 c}$ is the fastest during the whole gelation. 


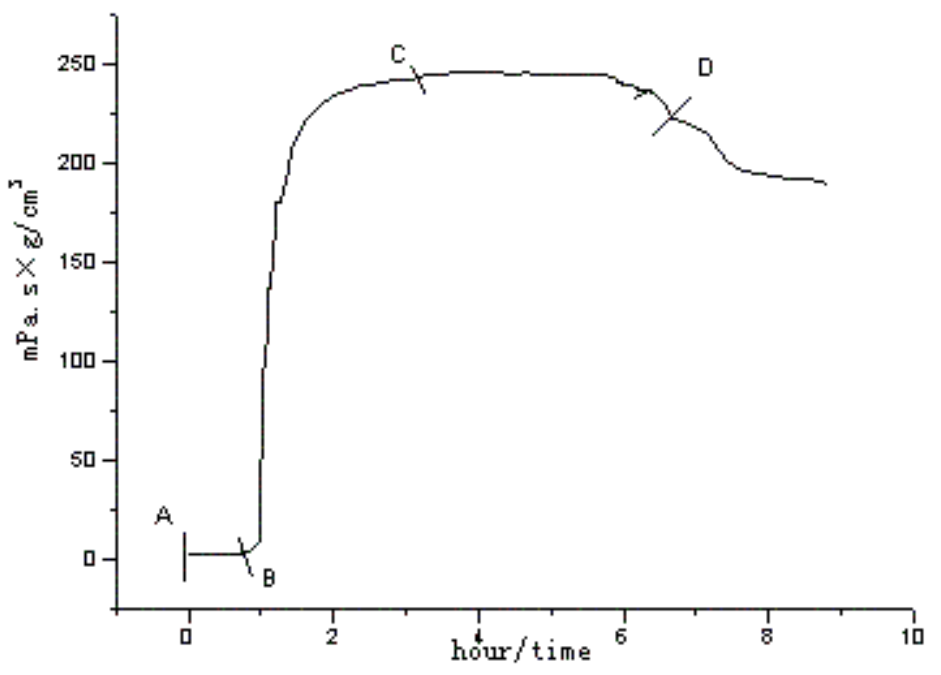

Figure 1. Plot of viscosity versus time for the copolymerization of the monomer 5a with PETA.

Table 2. Some results of copolymerization of active acrylate monomer 5 with PETA ${ }^{\mathrm{a}}$

\begin{tabular}{lllllllll}
\hline Monomer & \multicolumn{3}{l}{ Period of time (hour) ${ }^{\mathrm{b}}$} & \multicolumn{4}{c}{ Distribution and content of chlorine in foam(wt\%) } \\
\hline & A to B & B to C & C to D & Cl(t) & Cl(c) & Cl(b) & Cl(a) & Cl(th.) \\
\cline { 2 - 9 } & 0.94 & 2.42 & 3.36 & 14.98 & 15.87 & 15.56 & 15.47 & 19.34 \\
5b & 1.66 & 2.26 & 3.92 & 16.62 & 15.96 & 14.11 & 15.56 & 18.53 \\
5c & 0.44 & 2.44 & 2.88 & 1.21 & 1.38 & 1.53 & 1.37 & 18.53 \\
\hline
\end{tabular}

${ }^{\mathrm{a}}$ The copolymerization of monomer $\mathbf{5}$ with PETAwas carried out by using AIBN as initiator and dioxane as solvent at $70^{\circ} \mathrm{C}$. ${ }^{b}$ The period of time of polymer formation was monitored by viscometer (see Figure 1). ${ }^{\mathrm{c}}$ Data from element analysis: $\mathrm{Cl}(\mathrm{t})$ was the sample taking from the top of foam, $\mathrm{Cl}(\mathrm{c})$ was the sample taking from the center of foam, $\mathrm{Cl}(\mathrm{b})$ was the sample taking from the bottom of foam, $\mathrm{Cl}(\mathrm{a})$ was the average value of sample from the top, center and bottom of foam, $\mathrm{Cl}(\mathrm{th})$ was theoretical value of sample.

The foams were produced by the precipitation and desolvation of a gel prepared by copolymerization of the monomer 5 with PETA. The foams with special properties can only be produced when the solvent is removed in the absence of capillary forces in the gel pores, because even small capillary forces cause shrinkage and collapse of the gels network structure. Supercritical carbon dioxide possesses desirable physical properties, such as liquid-like densities and gas-like viscosities, so that its utilization provides a safer and more environmentally responsible alternative. Supercritical carbon dioxide drying eliminates surface tension and keeps the network structure of the gel from destroying to obtain the acrylate foam. During the drying process, methanol in the gel pores was replaced by carbon dioxide at conditions above the critical conditions of $\mathrm{CO}_{2}$, i.e. $304 \mathrm{~K}$ and $7.4 \mathrm{MPa}$. 
Table 2 shows some preliminary results for the distribution and content of chlorine in the foam by element analysis. The samples were collected from the top, the center and the bottom of foams respectively. The results indicate that the chlorine is distributed into the foam structure all over by chemical bond method. In addition, very excellent reactivity of $\mathbf{5 a}$ and $\mathbf{5 b}$ with PETA can afford the acrylate foams containing large content of chlorine. The content of chlorine in the foam for monomer $\mathbf{5 c}$ was so low. Perhaps, for this monomer $\mathbf{5 c}$ the copolymerization process is not takes place and the copolymer is not formed; or $\mathbf{5 c}$ may be homopolymerized to form low-polymer, and then washed out in followed solvent exchange process.

\section{Experimental Section}

General Procedures. Unless otherwise specified, all chemicals were of purity grade and used without further purification. Pentachlorophenol (PCPOH) was prepared from the reaction of its sodium salt with hydrochloric acid. Pentaerythritol tetraacrylate (PETA, inhibited with $350 \mathrm{ppm}$ MEHQ) were purchased from Aldrich. Azobis(isobutyronitrile) (AIBN) was recrystallized before use. Other chemicals were purchased from Shanghai Reagent Co. Solvents were purified by using the standard methods. ${ }^{1} \mathrm{H}$ NMR spectra were recorded on a Varian INOVA-400 spectrometer; the chemical shifts are indicated in $\delta$ value (ppm) downfield from internal TMS. Mass spectra were measured on a Finnigan MAT 4510 spectrometer. Melting points were measured on a XRC-1 melting point apparatus and are uncorrected. A Nexus 670 Infrared spectrometer was applied for recording spectra in KBr pellets or films. A NCGS-4 ultrasonic viscometer was used to record the viscosity of the reaction system and monitoring the progress of reaction. The content and distribution of chlorine in foams were determined by elemental analysis.

Synthesis of pentachlorophenyl acrylate monomer 5. To a cold stirred solution of PCPOH 2 $(8.78 \mathrm{~g}, 0.033 \mathrm{~mol})$ at $0{ }^{\circ} \mathrm{C}$ in ethyl acetate $(10 \mathrm{~mL}), \mathrm{DCC} 1(2.06 \mathrm{~g}, 0.01 \mathrm{~mol})$ was added. The mixture was allowed to stand in the freezer overnight during which time a crystalline material deposited. Cold petroleum ether (PE) $\left(10 \mathrm{~mL}, 0{ }^{\circ} \mathrm{C}\right)$ was added to the reaction mixture. The solid was filtered and washed with cold PE. Recrystallization from hot PE (150 mL) yielded $8.20 \mathrm{~g}$ $(80 \%) 3.3(1.005 \mathrm{~g}, 0.001 \mathrm{~mol})$ was dissolved in ethyl acetate $(10 \mathrm{ml})$ at room temperature and then the appropriate acrylic acids $4(0.001 \mathrm{~mol})$ was added. The reaction mixture was allowed to stand for another $24 \mathrm{~h}$. The resulting solid cake was converted to a finely divided suspension after addition of ethyl acetate $(10 \mathrm{~mL})$. The suspension was cooled in ice bath for $1 \mathrm{~h}$ and filtered. The residue was suspended in ethyl acetate $(5 \mathrm{~mL})$ again, filtered, and washed twice with $2 \mathrm{~mL}$ of ethyl acetate. The combined filtrate was evaporated to dryness under vacuum at room temperature. The residue was suspended many times with PE, and the filtrate was cooled overnight in refrigerator to remove trace of dicyclohexylurea (DCU). After the filtrate was 
evaporated to dryness, flash chromatography of the residue on silica gel by using PE as eluent gave the desired pure product 5 in $70-78 \%$ yield. All characteristic data see Table 1.

Copolymerization of 5 with PETA. PETA was copolymerized with loaded-chlorine acrylate monomer 5 by using AIBN as initiator. The copolymerization was carried out using Schlenk techniques in a dry nitrogen atmosphere and monitored in time by ultrasonic viscometer. A typical copolymerization procedure was described as follows: AIBN (25 mg) was weighed into a Schlenk tube. Then a solution of dioxane $(50 \mathrm{~mL})$ containing a mixture of PETA $(1.627 \mathrm{~g}$, $0.0043 \mathrm{~mol})$ and monomer $5 \mathrm{a}(0.873 \mathrm{~g}, 0.0026 \mathrm{~mol})$ was injected by a syringe. The tube was placed in a thermostatically controlled bath at $70{ }^{\circ} \mathrm{C}$. After a certain time the gels were formed.

Supercritical carbon dioxide drying of the gels. After the gelation was terminated, the gel was precipitated by immersion in methanol for 2-3 weeks to allow the solvent dioxane and the methanol to exchange completely. Subsequently, the gels was separated by filtration and washed with methanol. The pretreated gels were dried with supercritical carbon dioxide in a supercritical drying apparatus at a temperature of $40^{\circ} \mathrm{C}$ and a pressure of $10 \mathrm{MPa}$. The desired foams were obtained over a period of time.

\section{Conclusions}

We have developed a novel method to prepare the active acrylate monomers with chlorine by introducing successfully PCP group into the backbone of acrylate monomer. Copolymerization of the perchlorinated monomers with PETA was studied. The preliminary results indicated that the acrylate foams can be produced on supercritical carbon dioxide drying and the chlorine was distributed throughout the foam structure by chemical bound method. Currently, further characterization of the foams and their application in the fabrication of targets for laser-driven plasma physics experiments are underway.

\section{Acknowledgements}

This work was financially supported by National Natural Science Foundation of China (No. 20328203, 20272039) and National Key Laboratory of Laser Fusion (No.51480030203ZW0803).

\section{References}

1. Rosenberg, J. E.; Flodin, P. Macromolecules 1986, 19, 1543.

2. Rosenberg, J. E.; Flodin, P. Macromolecules 1987, 20, 1518.

3. Rosenberg, J. E.; Flodin, P. Macromolecules 1987, 20, 1522. 
4. Rosenberg, J. E.; Flodin, P. Macromolecules 1988, 20, 2041.

5. Falconer, J. W.; Golnazarians, W.; Baker, M. J.; Sutton, D. W. J. Vac. Sci. Technol. A 1990, $8,968$.

6. Apen, P. G.; Armstrong, S. V.; Moore, J. E.; Esinoza, B. F.; Gurule, V.; Gobby, P. L.; Williams, J. M. J. Vac. Sci. Technol. A 1993, 11, 1248.

7. Falconer, J. W.; Nazarov, W.; Horsfield, C. J. J. Vac. Sci. Technol. A 1995, 13, 1941.

8. Nazarov, W. Fusion Sci. Technol. 2002, 41, 193.

9. Falconer, J. W.; Nazarov, W.; Horsfield, C. J.; Sutton, D. W.; Rothman, S. D.; Freeman, N. J. J. Vac. Sci. Technol. A 1994, 12, 2798.

10. Faith, D.; Nazarov, W.; Horsfield, C. J. Fusion Science and Technology 2004, 45, 90.

11. Batani, D.; Benuzzi A, Boudenne, J. M.; Faral, B.; Hall, T.; Koenig, M.; Lower, T.; Muller, L.; Nazarov, W.; Temporal, M. J. Moscow Phys. Soc. 1998, 8, 257.

12. Batani, D.; Benuzzi, A.; Koenig, M.; Faral, B.; Temporal, M.; Nazarov, W.; Hall, T.; Granjoune, N. Control Fusion 1998, 40, 1567.

13. Takagi, M.; Norimatsu, T.; Yamanaka, T.; Nakai, S. J. Vac. Sci. Technol. A 1991, 9, 820.

14. Nazarov, W.; McGivern, P. G. Fusion Technol. 2000, 38, 110.

15. Nazarov, W. J. Moscow Phys. Soc. 1998, 8, 251.

16. Sutton, D. W. J. Vac. Sci. Technol. A 1987, 5, 2773.

17. Kovacs, J.; Kisfaludy, L.; Ceprini, M. Q.; Johnson, R. H. Tetrahedron 1969, 25, 2555.

18. Bodanszky, M.; Bodanszky, A. The Practice of Peptide Synthesis, $2^{\text {nd }}$ Edn.; Springer: Berlin, 1994; Vol. III, p 101.

19. Wang, X.-Y.; Wang, Q.; Huang, X.-Y.; Wang, T., Yu, X.-Q. ARKIVOC 2006, (xi), 148.

20. Kaji, A.; Hashimoto, K.; Kano, S. Nippon Kagaku Zasshi 1961, 82, 782.

21. Kaji, A.; Kano, S.; Hashimoto, K. Jap. Patent 38011612, 1963: Chem. Abstr. 1963, 59, 13883.

22. Matsuda, T.; Sugisawa, H.; Funae, Y.; Kondo, T.; Takatani, N. Jap. Patent 62063541, 1987.

23. Dong, J.-H.; Meng, W.-D.; Qing, F.-L. ARKIVOC 2004, (ix), 9.

24. Pittman, C. U.; Lawyer, K. R. Org. Coat. Plast. Chem. 1981, 44, 12.

25. Pittman, C. U. J. Appl. Polym. Sci. 1981, 26, 2403.

26. Potin, C.; Pleurdeau, A.; Bruneau, C. M. Angew. Makromol. Chem. 1986, 138, 75.

27. Gorchakova, V. A.; Budzan, B. I.; Andronova, N. A.; Kolokolov, B. N.; Virnik, A. D. Zhurnal Prikladnoi Khimii 1984, 57(5), 1121. 